REVIEW

\title{
Indeterminate colitis
}

\author{
M Guindi, R H Riddell
}

J Clin Pathol 2004;57:1233-1244. doi: 10.1136/icp.2003.015214

Indeterminate colitis (IC) originally referred to those 10$15 \%$ of cases of inflammatory bowel disease (IBD) in which there was difficulty distinguishing between ulcerative colitis (UC) and Crohn's disease (CD) in the colectomy specimen. However, IC is increasingly used when a definitive diagnosis of $U C$ or CD cannot be made at colonoscopy, in colonic biopsies or at colectomy. The diagnostic difficulties may explain the variably reported prevalence of IC. Clinically, most patients with IC evolve to a definite diagnosis of $U C$ or $C D$ on follow up. The role of ancillary tests in the distinction of UC from CD is reviewed. The low sensitivity of serological markers limits their usefulness. Other tests include upper endoscopy and magnetic resonance imaging. The definition of IC may not be a purely histological one derived from resected specimens, but rather a clinicopathological one. This review offers some personal observations and viewpoints, and proposes an approach to some of the relatively more esoteric combinations of findings.

See end of article for authors' affiliations

Correspondence to: Dr M Guindi, Department of Pathology, Toronto General Hospital, 200 Elizabeth Street, 4 th floor, Room EC4-305, Toronto, Ontario, Canada, M5G 2C4; maha.guindi@uhn. on.ca

Accepted for publication 1 June 2004
$\mathrm{T}$ he distinction between ulcerative colitis (UC) and Crohn's disease (CD) has major implications, including the choice of medical treatment, timing of surgery, prognosis, and disease course. In addition, there is a need for surveillance in UC, and possibly also in Crohn's colitis, because it may have a similar risk to UC. The distinction between UC and CD usually determines whether an ileal pouch anal anastomosis (IPAA) is offered to the patient, and correlates with the occurrence of morbidity and complications from pouch and pouch failure. Because there can be overlapping features of UC and $\mathrm{CD}$ in the colons of some patients, the term indeterminate colitis (IC) was coined in an attempt to classify these entities more effectively. Patients with indeterminate colitis appear to have a higher rate of pouch failure and longterm complications than those with UC.

"The distinction between ulcerative colitis and Crohn's disease has major implications, including the choice of medical treatment, timing of surgery, prognosis, and disease course"

This review of IC will deal with its original definition and features, how it evolved over time, and the controversies associated with this evolution. The incidence, prevalence, and clinical evolution of IC will be followed by a detailed discussion of the settings that give rise to the diagnosis of IC, and the problems and pitfalls associated with them, including variations of inflammatory bowel disease (IBD), both UC and colitis of CD, which can be mistaken for IC. These include the more common features such as IBD in the fulminant or refractory phase, but also IBD in the chronic phase, and the effects of treatment on the histology of IBD, especially UC, which can result in pronounced focality, relative rectal sparing, and focal healing. The issues of interobserver variability in the diagnosis and classification of IBD and colitis as a result of causes other than UC or CD are discussed. Recent developments, and the usefulness of ancillary tests that are potentially helpful, in the further classification of IC are summarised, with special reference to serological markers and the role of upper endoscopy. The outcome of ileoanal pouch construction in IC is also discussed.

\section{DEFINITIONS AND FEATURES}

UC characteristically affects the rectum, involves the colon contiguously and symmetrically, and the disease is more severe distally. Crohn's colitis is not continuous or symmetrical, and need not involve the rectum. ${ }^{1-5}$ In 1978, Price introduced the term IC to refer to a subgroup of approximately $10-15 \%$ of IBD cases in which there was difficulty in distinguishing between UC and $\mathrm{CD}$ in the excised colectomy specimen because the features of typical severe UC were replaced by deep ulcers-often with knife-like fissures-relative rectal sparing, and transmural inflammation. ${ }^{6}$

Table 1 summarises the histological features and incidence of discriminating attributes in Price's UC, CD, and IC groups.

Idiopathic granulomas were only present in CD. Typical CD fissuring-type ulcerations (mostly single and narrow) were present in 13\% of cases of IC, but the other histological features of these IC cases were reportedly so atypical that a diagnosis of CD could not be justified. A second form of fissuring ulceration, V shaped clefts, usually multiple, was present in $60 \%$ of cases of IC, and appeared to be a feature of any form of fulminant colitis. Transmural lymphoid aggregates, a feature accepted as very important in the diagnosis of CD, was present in $6 \%$ of cases of IC,

Abbreviations: ASCA, anti-Saccharomyces cervisiae antibodies; ASLC, acute self limited colitis; CD, Crohn's disease; DDAC, diverticular disease associated colitis; $I B D$, inflammatory bowel disease; IC, indeterminate colitis; IPAA, ileal pouch anal anastomosis; NSAID, nonsteroidal anti-inflammatory drug; P-ANCA, perinuclear antineutrophil cyłoplasmic antibodies; UC, ulcerative colitis 
Table 1 Incidence of discriminating histological features in UC, CD, and IC*

\begin{tabular}{|c|c|c|c|c|c|}
\hline & \multirow[b]{2}{*}{ IC } & \multicolumn{2}{|l|}{$C D$} & \multicolumn{2}{|l|}{ UC } \\
\hline & & Urgent surgery & Elective surgery & Urgent surgery & Elective surgery \\
\hline Granulomas & 0 & 2 & 23 & 0 & 0 \\
\hline \multicolumn{6}{|l|}{ Fissures } \\
\hline Typical CD & 4 & 2 & 12 & 0 & 0 \\
\hline V shaped clefts & 18 & Not given & Not given & Not given & Not given \\
\hline Transmural inflammation & 28 & 2 & 30 & 20 & 0 \\
\hline $\begin{array}{l}\text { Normal goblet cells, regular gland pattern, and focal mucosal } \\
\text { inflammation }\end{array}$ & 20 & 2 & 27 & 5 & 2 \\
\hline $\begin{array}{l}\text { Goblet cell depletion, glandular irregularity, } \pm \text { diffuse mucosal } \\
\text { inflammation }\end{array}$ & 10 & 0 & 3 & 25 & 28 \\
\hline \multicolumn{6}{|l|}{ Feature suggestive of acute disease \pm dilatation } \\
\hline Myocytolysis and capillary congestion & 21 & 0 & 0 & 22 & 0 \\
\hline V shaped clefts & 18 & 0 & 0 & 6 & 0 \\
\hline
\end{tabular}

but were poorly developed. Transmural inflammation was present in most cases of IC, but was only related to areas of severe ulceration. The mucosal glandular pattern/architecture and goblet cell population were obscured or modified by extensive ulceration in the IC cases. The islands of surviving mucosa were only mildly inflamed, with a regular glandular pattern and preserved goblet cells-features that favoured $\mathrm{CD}$, or disease in mucosa that was previously unaffected by the disease process, conceivably including fulminant infections by organisms that were not identified on routine stool culture. However, these features were present in cases where the cumulative evidence was unclear, and two thirds of these proved to be UC on subsequent follow up in Price's series. ${ }^{7}$

"The presence of scattered mononuclear inflammatory cells in the muscularis propria adjacent to ulceration was regarded as a non-specific response, and not a discriminating feature between ulcerative colitis and Crohn's disease"

Approximately a half of the cases of IC described by Price ${ }^{6}$ had uneven disease that fell into two patterns, both of which would normally bias towards CD (table 2).

In the subgroup with relative rectal sparing, the description of the rectal mucosa was not very detailed. The rectal mucosa was described as being "mildly abnormal" on histological examination in all seven cases. In the three judged to be probably UC, glandular irregularity was noted. In the remainder of the IC cases with relative rectal sparing, the inflammation was described as mild, without specific reference to the presence or absence of basal plasmacytosis or architectural distortion. The second pattern was of intermittent ulceration and superficially resembled the skip lesions of $\mathrm{CD}$.

Table 2 Findings in uneven disease pattern in indeterminate colitis from Price ${ }^{6}$

\begin{tabular}{|c|c|c|}
\hline & \multicolumn{2}{|l|}{ Uneven pattern $(n=14)$} \\
\hline & $\begin{array}{l}\text { Relative rectal sparing } \\
\text { group }\end{array}$ & $\begin{array}{l}\text { Intermittent ulceration } \\
\text { group }\end{array}$ \\
\hline $\begin{array}{l}\text { Number of cases } \\
\text { UC more likely } \\
\text { CD more likely } \\
\text { Rectal mucosa } \\
\text { Mucosa between } \\
\text { ulcers }\end{array}$ & $\begin{array}{l}7 \text { cases } \\
3 \text { cases } \\
2 \text { cases } \\
\text { Mildly abnormal } \\
\text { Not specifically } \\
\text { mentioned }\end{array}$ & $\begin{array}{l}7 \text { cases } \\
3 \text { cases } \\
2 \text { cases } \\
\text { Not specifically mentioned } \\
\text { Mild inflammation in all } \\
\text { cases }\end{array}$ \\
\hline
\end{tabular}

Lee et al found IC in 16\% of colectomy specimens. ${ }^{8}$ Some of the features of IC they described are similar to those of Price et al, such as normal or minimally altered colonic mucosa adjacent to or between ulcers and the absence of granulomas. They described deep slit-like fissures in the IC cases, but did not specifically describe the $\mathrm{V}$ shaped cleft-like fissures seen in IC by Price, although these may just be synonyms. They also emphasised that the term "transmural inflammation", a feature of $\mathrm{CD}$, is loosely used and that accurate definition of this criterion removes much of the difficulty from the differential diagnosis of IBD. They defined transmural inflammation as lymphocytes in an aggregated pattern in all layers of the colon, including the serosa. The presence of scattered mononuclear inflammatory cells in the muscularis propria adjacent to ulceration was regarded as a non-specific response, and not a discriminating feature between UC and CD. Their IC cases contained scattered non-aggregated inflammatory cells involving the full thickness of the bowel wall, but only in those areas deep to ulceration. Unlike Price's series, all their patients had an acute onset and required urgent colectomy after a short period of unsuccessful medical management. ${ }^{8}$

\section{THE EVOLUTION OF IC}

The term IC is increasingly being used clinically and histologically in patients with some form of IBD in whom a definitive diagnosis of either UC or CD has not been made, either on colonoscopy or colonic biopsy before colectomy. IC may not be a purely histological entity, but rather a

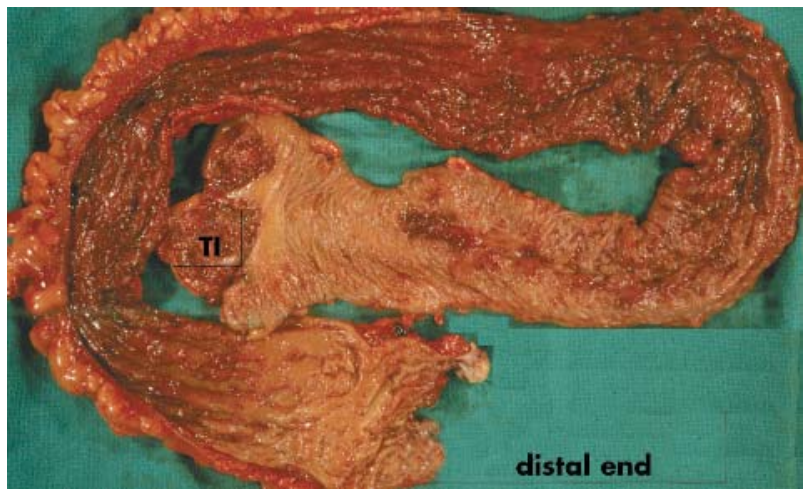

Figure 1 Relative distal sparing. Colectomy specimen showing less congested and less diseased appearing mucosa at the distal end of the colon, compared with the erythematous ulcerated colonic mucosa seen more proximally. In the right colon, the transition from normal to diseased mucosa is irregular and patchy. 
clinicopathological one, whereby the label is applied to indeterminate disease in the colectomy specimen (not in the preceding biopsies), and may remain as such, or be further refined into UC or CD as clinical events warrant. After correlating the pathology, preoperative clinical information, and radiological findings of 46 patients with a diagnosis of IC, Wells et al reclassified 46 patients from the original Price series with IC as follows: 19 patients classified as probable $\mathrm{CD}, 11$ classified as probable UC, whereas 16 retained the diagnosis of IC ( $40 \%$ were reclassified as CD). Over a median follow up of 10 years, the patients in the last group continued to be classified as IC, and evidence of CD was not found despite many clinical investigations. ${ }^{7}$

Even if the definition were standardised, in practice, the patients carrying a diagnosis of IC may be a heterogeneous group. Some may have colitis that cannot be subclassified into UC or CD (therefore labelled IC) despite investigations at referral centres by dedicated multidisciplinary teams of gastroenterologists, pathologists, and surgeons. Others, also designated as IC in less specialised centres or practices, may well have UC or CD if investigated by a more expert team.

\section{FACTORS LEADING TO A DIAGNOSIS OF IC}

It can be difficult to distinguish UC from $C D$ in cases of fulminant or refractory IBD, the chronic phase of IBD, when unusual patterns are seen in otherwise typical UC, in treated cases of IBD, and in the earliest stages of IBD. When the distinction between UC and CD becomes difficult, a diagnosis of IC is rendered. In addition, some patients may not have
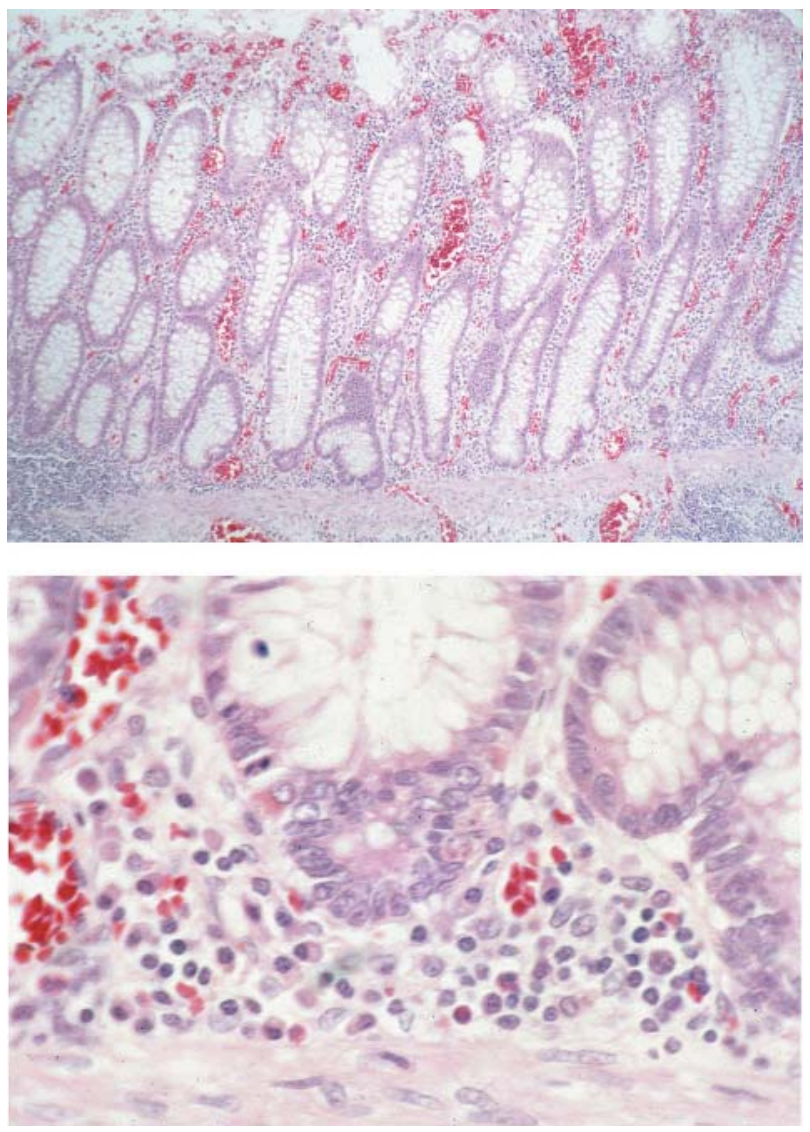

Figure 2 Residual mucosa in a colectomy specimen from fulminant inflammatory bowel disease. (A) Mucosal changes are minimal, without mucin depletion, and with only slight architectural distortion. (B) Mild inflammation only with basal lymphoplasmacytosis (haematoxylin and eosin stain).
IBD at all, and other possible aetiologies may need to be considered.

The difficulty in distinguishing UC from CD, thereby leading to a diagnosis of IC, is related to several confounding factors, as detailed below.

\section{IBD in the fulminant or refractory phase}

Some of the gross and microscopic features that are useful in distinguishing the two diseases in the chronic state are common to both in the fulminant or refractory phase. For example, relative rectal sparing (fig 1), intermittent ulceration, a regular glandular pattern, and lack of mucin depletion (fig 2)-features that in classic teaching would sway the evidence in favour of $\mathrm{CD}$-are seen in some indeterminate cases in Price's series ${ }^{6}$ that subsequently proved to be UC, or that lacked other evidence of CD. It has been surmised that the diagnosis of IC is more likely to follow the examination of a severely diseased colectomy specimen. ${ }^{9}$ In one series, fulminant disease was more common in patients with IC compared with either UC or CD, despite similar rates of immunosuppression. ${ }^{10}$ However, it should be noted that the inclusion of specimens from colectomy performed for fulminant colitis in a series selects for IC, as described by Price $^{6}$ - the histological distinction of UC from CD is more difficult in the fulminant setting because the histological features overlap. It has been argued that intensive steroid treatment for fulminant disease may lead to the histological appearance of IC. ${ }^{10}$ Histopathological evaluation alone has limitations in the accurate classification of fulminant IBD. Granulomas and transmural lymphoid hyperplasia/aggregates, especially when not in areas of ulceration, appear to be the two most specific indicators of CD in colectomy specimens from patients with fulminant colitis. ${ }^{11}$ Poorly formed microgranulomas may be good indicators of CD, although when an aggregate of histiocytes becomes a granuloma is highly subjective. Some have used five histiocytes to define microgranulomas - that at least provides some objectivity. ${ }^{12}$ Greater emphasis should perhaps be placed on their identification. ${ }^{11} 13$ Isolated giant cells and well defined epithelioid granulomas distant from crypts do not, as a rule, occur in UC, and hence their presence in a colonoscopic biopsy showing features of chronic IBD is a strong pointer towards the diagnosis of CD. Crypt associated giant cells and granulomas can occur in UC (fig 3), and in themselves are unreliable features for the discrimination between CD and UC. $^{12}$ A pathological review of the original histological diagnoses in Swan's series suggests that the reluctance to commit to a diagnosis of UC by some was based on the presence of features that are considered to be characteristic of

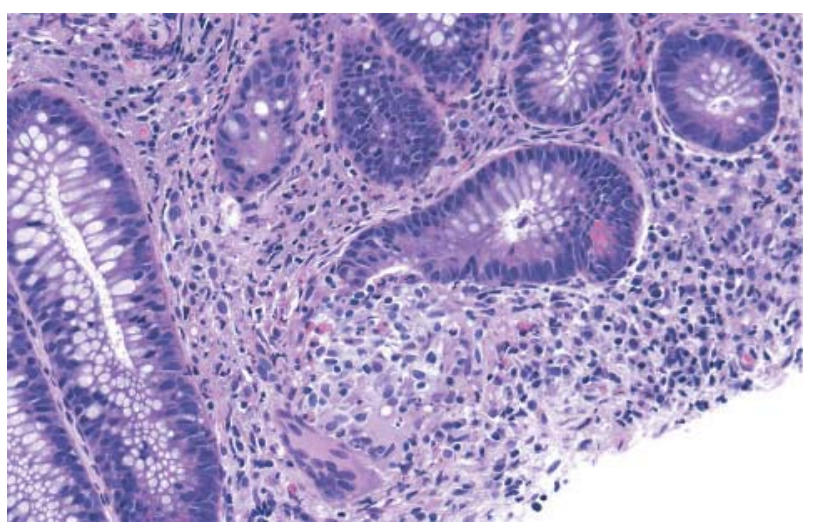

Figure 3 Crypt associated giant cells and granulomas. Note the associated ruptured crypt. The lesion forms as a reaction to extravasated mucin (haematoxylin and eosin stain). 
the fulminant nature of IBD, such as transmural inflammation and fissuring ulceration, and which may superficially be reminiscent of $\mathrm{CD} .{ }^{11}$ Macroscopic examination appears to have rather limited value in differentiating UC from CD in the fulminant state. ${ }^{11}$ In the fulminant colitis specimen, linear ulceration (fig 4) and fissures are relatively common, regardless of the precise cause, and are not specific indicators of $\mathrm{CD}$.

\section{"The histological distinction of ulcerative colitis from Crohn's disease is more difficult in the fulminant setting because the histological features overlap"}

\section{IBD in the chronic phase}

Even in the chronic phase, the classic endoscopic and histological features of UC do not always conform to the traditional rules, which may lead to confusion with CD, change the diagnosis to Crohn's colitis, or lead to a diagnosis of IC. In practice, this may occur at the time of examination of endoscopic biopsies in the non-fulminant setting, and not just upon examination of colectomy specimens resected for fulminant disease. ${ }^{14-18}$ There are sequential biopsy changes in the mucosa of UC that occur with time, and pertain to disease distribution and extent. In one study of patients with UC, who had a median follow up of seven years, ${ }^{16}$ these changes included: development of non-diffuse distribution, finding of normal rectal mucosa both endoscopically and histologically, either at the beginning or later on in the course of the disease, variation in extent of involvement over time, and lack of an endoscopic-histological correlation. The presence of some of these features in biopsy specimens may not fit with the rest of the cumulative evidence for UC in a given case, or may render the cumulative evidence equivocal, such that a diagnosis of IC is made. One caveat to this study is that because several biopsy sets from the same patient had been taken over time, some of the variability in histological and endoscopic appearances may have been related to the effects of treatment and were not necessarily a "natural" evolution of extent and distribution. Particularly in quiescent disease, some pathologists may be less inclined to offer a specific diagnosis other than IBD when the pattern and distribution of activity that would favour one or other diagnosis cannot be assessed. A diagnosis of quiescent IBD based primarily on architectural distortion and its distribution is not the same thing as IC. When IBD is inactive, only minimal histological changes are found, making a histological differential diagnosis of CD and UC difficult, ${ }^{19-21}$ and also making it difficult to distinguish from infection, especially retrospectively. This raises the issue of whether one can or should lend

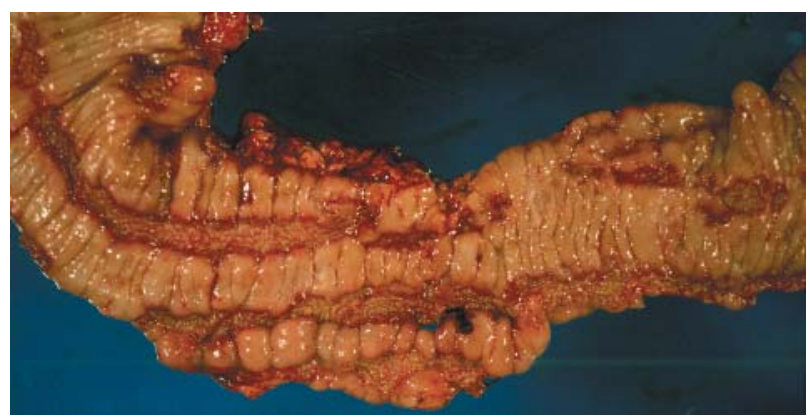

Figure 4 Colectomy specimen from a patient with refractory inflammatory bowel disease. Longitudinal ulcers are present and are not specific for Crohn's disease. Note the almost normal or minimally involved mucosa. more credence to the features at first presentation or those seen after treatment, when the acute phase has passed.

\section{Unusual patterns in UC}

Some patients with either subtotal or left sided colitis may show patchy, mild, caecum (caecal patch), and/or periappendiceal orifice and/or ascending colon chronic active inflammation, which is separated from the distal disease by normal intervening colonic mucosa, giving the false impression of a skip area, and therefore CD. ${ }^{22-24}$ In one series of 20 patients with established left sided UC, six showed a sharp demarcation between affected and unaffected portions of colon, whereas 14 showed a more gradual transition. ${ }^{22}$ The area of transition may appear somewhat patchy (fig l) and give the false impression of skip lesions. Furthermore, three quarters of this last group of patients showed an area of inflammation in the caecum, primarily in the periappendiceal mucosa, which was separate from the distal inflamed segment. The natural history of UC in patients with a caecal patch suggests that patchy right sided inflammation in patients with left sided colitis has little clinical relevance, but should be recognised by pathologists to prevent a false diagnosis of $\mathrm{CD}$ in this setting. ${ }^{25}$ Discontinuous involvement of the appendix in 13 of 62 cases of UC was first described by Davison and Dixon. ${ }^{26}$ Others subsequently reported this observation. In the series of Groisman et al there were two cases with limited left sided involvement combined with appendiceal involvement. ${ }^{27}$ The appendiceal involvement is mucosal but can be quite severe; however, it appears not to produce clinical symptoms of appendicitis (or these are masked by those of the underlying colitis).

\section{"Definite diagnostic criteria for backwash ileitis have not been determined, especially those to delineate the extreme end of its spectrum"}

Backwash ileitis represents a mild (but occasionally severe and extensive) degree of active inflammation in the distal few centimetres of terminal ileum in some patients with severe pancolitis. It is presumed to be secondary to the reflux of colonic contents. ${ }^{28}$ When present and exaggerated, it may raise the differential diagnosis of $\mathrm{CD}$. However, $\mathrm{CD}$ more typically involves longer segments of the distal ileum, and may show other features of CD, such as granulomas. Definite diagnostic criteria for backwash ileitis have not been determined, especially those to delineate the extreme end of its spectrum, but conventionally diffuse mucosal disease in continuity with severe colonic disease is accepted, as long as no other features of CD or other diseases are present. Despite earlier reports that backwash ileitis was not an important risk factor for the development of pouchitis, ${ }^{29}$ one subsequent study suggested that patients who are preoperatively assessed to have ileal disease appear to be at greater risk for the development of pouchitis. ${ }^{30}$

\section{Effects of treatment on histology of IBD}

Some of the changes described above may be related to the effects of treatment. ${ }^{151631}$ A study that prospectively evaluated rectal histology in patients enrolled in a 5-aminosalicylic acid enema treatment trial found that seven of 11 patients with UC treated with 5-aminosalicylic acid had one or more biopsies that showed histologically normal rectal mucosa. ${ }^{32}$ In two of the three patients treated with placebo, one or more biopsies were normal. Focality of healing, including rectal healing, occurs in all forms of treatment. Using sequential endoscopic biopsies, Kim et al have reported endoscopic and histological patchiness of inflammation and rectal sparing in 59\% of well documented patients with UC over time. ${ }^{31}$ The Leuven group (Geboes and Dalle) performed 
a literature review of clinical drug trials in IBD and the effects of various drugs on the microscopic features of $\mathrm{CD}^{33}$ Diagnostic microscopic features and the features characteristic of disease activity vary with time and treatment. The more recently developed drugs (especially the immunomodulators azathiaprine and infliximab ${ }^{22}{ }^{34}$ ) used for CD can induce mucosal healing. Medical treatment can have profound effects on mucosal histology, but the effect is highly variable. It depends on time, the type of treatment (whether topical or systemic), the intensity of the inflammation, the severity of the lesion, and probably several other factors that are unknown at the present time. In all drug studies involving CD it must be remembered that there is about a $30 \%$ response rate for any form of treatment (a placebo response), so that genuine responses must result in considerably greater degrees of therapeutic responsiveness.

Iatrogenic inflammatory changes that may be confused with IBD may represent the effects or bowel preparation compounds administered before lower endoscopy. Sodium phosphate, a commonly used oral cathartic agent, causes aphthoid ulcers or focal active colitis in the colon and rectum that might lead to an endoscopic diagnosis of $\mathrm{CD}$, especially in quiescent IBD. ${ }^{35}$

\section{Early stage IBD}

It has been shown that the microscopic features used for the diagnosis of IBD are often not present in the very early stage of disease, ${ }^{36}$ especially in children. ${ }^{37}{ }^{38}$ For example, children may show relative, or complete, rectal sparing or even patchiness of disease at initial presentation before treatment. ${ }^{36} 373^{40}$ In a study of rectal and sigmoid colon biopsies from untreated paediatric patients with UC evaluated shortly after the onset of symptoms, approximately $50 \%$ of patients had completely normal biopsy results and two had an endoscopically normal rectum and sigmoid. ${ }^{39}$ Compared with adults, the paediatric group showed significantly fewer patients with chronic active disease, and more patients with microscopic skip areas and relative rectal sparing. ${ }^{41}$ Atypical features of UC in children at initial presentation such as the absence of features of chronicity, mild active disease, and microscopic skip areas creates several dilemmas: they do not exclude the possibility of UC, may lead to a diagnosis of CD in error, and make it difficult to discern IBD from infection. A diagnostic re-evaluation of patients in the southeastern Norway study has shown that in 33\% the presence of IBD was either excluded or strongly questioned during follow up. ${ }^{42}$

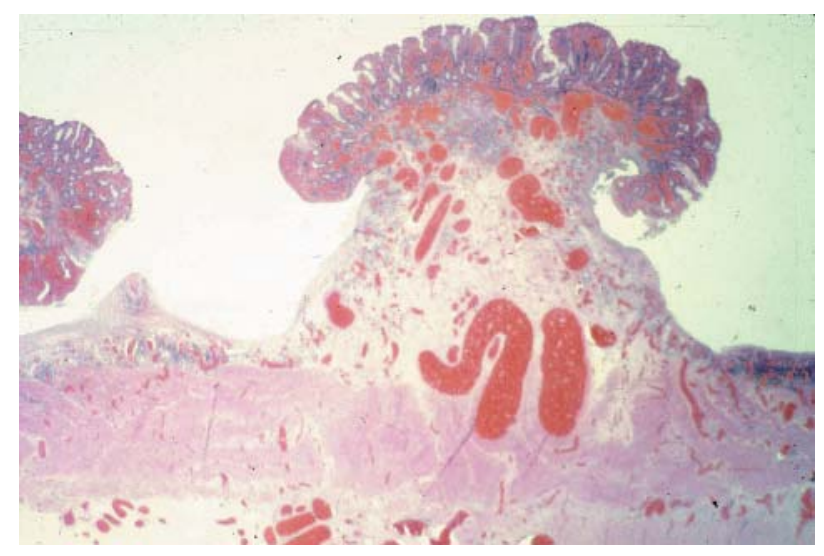

Figure 5 Deep wide ulcers that can be seen in fulminant colitis of any aetiology and can be associated with surrounding, sometimes transmural, inflammation in their vicinity but without lymphoid aggregates (Haematoxylin and eosin).

\section{Colitis other than UC and CD, including fulminant colitis of any aetiology}

When there is no previous clinical diagnosis, or in a first attack that is fulminant, colitis may have features that are found commonly, irrespective of the aetiology. These include deep ulcers with associated non-aggregated polymorphous transmural inflammation (fig 5), residual mucosa with mild inflammation, and no features of chronicity from preexisting IBD such as architectural distortion, minimal ulcers at bases of innominate grooves (fig 6), and pronounced capillary vasodilatation and haemorrhage. ${ }^{1}$ In this situation, some cases presenting as fulminant colitis may not easily fit into either UC or CD because the underlying disease has another cause. If the diagnosis of IC is made by default, it would inadvertently or erroneously imply that the underlying disease is IBD (UC or CD), or an intermediate form between them.

It has been proposed that the use of the term "fulminant colitis of uncertain aetiology" rather than IC should be used when there is no clear evidence of either previous UC or features that allow a diagnosis of $\mathrm{CD}$ to remove one area of ambiguity. ${ }^{1}$

\section{"In acute infectious colitis, a pathogen cannot be grown in up to $50 \%$ of patients, and a similar proportion of culture negative infections may also occur in fulminant colitis"}

The differential diagnosis in such cases should include IBD, in addition to forms of colitis that can occur as fulminant disease, such as colitis caused by infection (for example, Clostridium difficile, salmonellosis, shigellosis, and Escherichia coli) and drugs, such as non-steroidal anti-inflammatory drugs (NSAIDs). ${ }^{1}$ In acute infectious colitis, a pathogen cannot be grown in up to $50 \%$ of patients, and a similar proportion of culture negative infections may also occur in fulminant colitis. Even in a patient with known UC or CD, fulminant colitis may be infection mediated.

NSAIDs have been linked to flares of IBD and to triggering a first episode of IBD. ${ }^{43}$ NSAIDs can cause non-specific colitis or exacerbate pre-existing colonic disease. ${ }^{44}$

There is no clear agreement on the diagnostic criteria for excluding IBD, especially in patients with colonic disease only. ${ }^{45}$ Histological features can sometimes differentiate

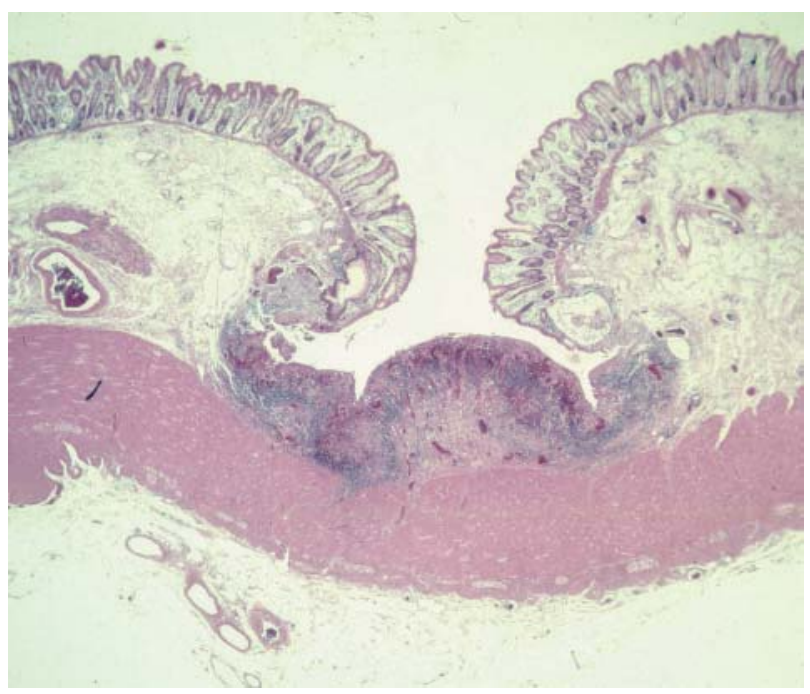

Figure 6 Aphthoid-like ulcers at the bases of innominate grooves may be seen in fulminant colitis of any aetiology (haematoxylin and eosin stain). 
between acute self limited colitis (ASLC) and UC, ${ }^{46}{ }^{47}$ and also between IBD and other types of colitis, including ischaemia and infection. ${ }^{48}$ Basal plasmacytosis in the lamina propria distal to the ileocaecal valve region (where it is normal) and mucosal distortion differentiate first and recurrent attacks of UC from cases of ASLC, ${ }^{46}{ }^{47}$ with the caveat that focal cryptitis during the resolving phase of ASLC could be confused with similar lesions in CD. However, as discussed above, the histological features used for the diagnosis of IBD are often absent in the very early stages of the disease.

Diverticular disease can mimic IBD in a variety of ways. Diverticular disease can be associated with chronic inflammatory changes of the luminal mucosa of the colon in the segments involved by diverticula, usually the sigmoid colon, known as diverticular disease associated colitis (DDAC). The inflammatory pattern ranges from mild non-specific inflammation, with or without mild crypt distortion, to a pronounced chronic active colitis picture that may mimic CD or UC, with cryptitis and crypt abscesses present. ${ }^{49}{ }^{50}$ The CD-like variant of DDAC can be seen in patients who have no evidence of $\mathrm{CD}$ elsewhere in the gastrointestinal tract, and appears to represent a local chronic inflammatory reaction to complicated (perforated and resolving) diverticulitis. This CD-like variant exhibits all the histological features of CD, including transmural lymphoid aggregates. ${ }^{51-53}$ Inflammatory masses related to diverticular disease may be associated with IBD-like changes of the overlying luminal mucosa. ${ }^{54}$ Diverticular disease may be associated with prolapsing mucosal polyps of the colon, especially the sigmoid colon, which may be confused endoscopically with inflammatory polyps of IBD..$^{55}$ Any combination of the above features may be present in biopsies or resections from a patient with diverticular disease. If the pathologist is unaware of the presence of diverticula when interpreting biopsies in this setting, or if the pathologist does not recognise the potential reaction patterns associated with diverticular disease, an erroneous diagnosis of IBD can be made. Classification of the "IBD" into UC or CD in this setting is difficult and may result in an inadvertent diagnosis of IC. The finding that in some patients diagnosed with DDAC the disease subsequently evolved into distal UC, despite initial normal rectal biopsies, ${ }^{49}$ further confounds the situation.

In ischaemic colitis, the endoscopic and clinical findings can overlap with UC or CD, making the distinction difficult, ${ }^{56}{ }^{57}$ especially in the elderly. It may be difficult, if not impossible, to distinguish ischaemia from IBD histologically. ${ }^{1}$ Segmental distribution may occur in ischaemia, similar to CD. Submucosal oedema and haemorrhage occur in ischaemia and in $\mathrm{CD}$, leading to cobblestoning and thumbprinting, which may be seen in both. Low flow ischaemia may be impossible to distinguish from UC if diffuse and with rectal involvement, or from CD if focal. Mucosal fibrosis and telangiectasia of capillaries, if present, favour ischaemia, but haemosiderin laden macrophages may be seen in ischaemic colitis and in IBD. Ischaemic proctosigmoiditis has been reported without proximal colonic involvement. ${ }^{58}$ Biopsies may show a non-specific ulcer base similar to the nonspecific ulcer base seen in IBD related ulcers and erosions.

Finally, mass lesions of diverse aetiologies, such as primary and metastatic malignancy, endometriosis, and pneumatosis, may cause overlying mucosal changes that closely mimic IBD in mucosal biopsies ${ }^{54}$; however, the clinical context in such cases would not be consistent with IBD.

\section{INTEROBSERVER VARIABILITY (OBSERVER BIAS)}

There is interobserver disagreement in the diagnosis and classification of colonic IBD, which can result in a change of diagnosis that can include either more or less patients in that category. Some pathologists are more sensitive to features that might be found in $\mathrm{CD}$, and are also more prepared to accept a larger range of changes in UC, thereby diminishing the number of patients with IC or CD. ${ }^{59-61}$ Theodossi et al found that the range of agreement among 10 observers with a special interest in gastrointestinal pathology was $65-76 \%{ }^{60}$ The best agreement was when discriminating between normal slides and those of confirmed IBD cases. Using $\kappa$ statistics, Farmer et al compared the histological diagnosis of colonic IBD made by board certified, university affiliated pathologists versus that made by a specialist gastrointestinal pathologist, and found significant interobserver variation. ${ }^{61}$ The gastrointestinal pathologist's diagnoses differed from the initial diagnoses in $45 \%$ of surgical specimens and 54\% of biopsy specimens. Forty three per cent of cases initially diagnosed as UC were changed to CD or IC, whereas $17 \%$ initially diagnosed as CD were changed to UC or IC. This study raises several interesting observations. Whereas no diagnosis of IC was made by the general pathologists, the gastrointestinal pathologist reclassified the IBD as IC in approximately $24 \%$ of cases. It would be tempting to assume that in the hands of a gastrointestinal pathologist fewer cases of IBD would fall into the IC category. However, this does not appear to be the case. One explanation may be the more in depth knowledge and/or experience of the gastrointestinal pathologist with regard to the overlapping features between $\mathrm{CD}$ and UC, and familiarity with the features of fulminant disease and with treatment effects that result in focality or some degree of rectal sparing. The number of paraffin wax blocks for each surgical colectomy specimen varied greatly, between two and 14. Sampling of colectomy specimens did not appear to be uniform, and therefore may have affected the ability of the pathologist to classify IBD. The adequacy of sampling in the IBD biopsy sets in Farmer's study was not clear. Inadequate sampling can potentially increase the frequency of the diagnosis of IC, both in colectomy resection specimens and in IBD biopsies.

\section{"Some pathologists are more sensitive to features that might be found in Crohn's disease (CD), and are also more prepared to accept a larger range of changes in ulcerative colitis, thereby diminishing the number of patients with indeterminate colitis or $\mathrm{CD}^{\prime \prime}$}

The contributions of the extent of sampling (multiple and single biopsies), expert status, brief exposure to guidelines, and the use of particular evidence based diagnostic criteria to the accuracy and reproducibility of the diagnosis of IBD were studied at an international workshop. ${ }^{62}$ Expert and nonexpert international diagnostic histopathologists attended a workshop. Sixty cases with full follow up were reviewed, blinded, in two rounds. Diagnoses were made on rectal biopsies and then full colonoscopic series. Experts correctly identified only $24 \%$ of CD cases (non-experts, $12 \%$ ) from the rectal biopsies (many were normal). This improved to 64\% (non-experts, 60\%) with the full series. The accuracy of the diagnosis of UC also improved slightly with the full seriesfrom $64 \%$ to $74 \%$ overall. Experts had a similar (moderate) level of agreement and accuracy compared with non-experts. A full colonoscopic series gave more accurate diagnosis than a rectal biopsy.

Tanaka et al found that interobserver agreement for histological features that distinguish IBD from other forms of colitis, and UC from CD (crypt atrophy, crypt distortion, basal plasmacytosis, grade of mononuclear infiltration, Paneth cell metaplasia, grade of mucin depletion, mucin preservation, focal or diffuse mononuclear infiltration, and epithelioid granulomas) was fair to good when assessed by $\kappa$ statistics. ${ }^{2}$ Agreement was maintained for all the examined 
features except Paneth cell metaplasia. It would appear that interobserver agreement regarding the identification of individual histological features is sufficiently reproducible, whereas interobserver disagreement regarding the synthesis of the overall diagnosis may be significant. There are differences in the identification and interpretation of lymphoid aggregates and equivocal granulomas among pathologists, which may lead to shifting the diagnosis from CD to IC, or vice versa. ${ }^{11}{ }^{12}$ Systematised protocols and checklists for endoscopic biopsies and colectomy specimens may help to improve interobserver variability.

\section{INCIDENCE/PREVALENCE AND CLINICAL EVOLUTION OF IC}

Factors that may contribute to variation in the prevalence of IC include interobserver variation in histological interpretation, making the diagnosis of IC based on the evaluation of endoscopic biopsy sets not colectomy specimens, determining prevalence based on colectomy specimen diagnosis with or without interval follow up during which CD may declare itself, and variable sampling of colectomy specimens. The study by Swan et al exemplifies several of these factors. ${ }^{11}$ Of 67 patients with IBD who underwent colectomy, 40 were diagnosed as UC, 16 as CD, and 11 as IC immediately after surgery by several pathologists. The prevalence of IC changed to nine of 67 upon review of the cases by the study pathologist, and to six of 67 after a mean follow up period of 43 months. There appears to be reluctance among general pathologists to make a diagnosis of Crohn's colitis in the absence of granulomas. ${ }^{61}$ This may impact upon the prevalence of IC in study series of colectomy or biopsy sets, and adds to interobserver variation.

The prevalence of IC diagnosed preoperatively may increase or decrease after surgery and evaluation of the colectomy specimen; it fell from $13 \%$ to $4 \%$ in one study ${ }^{63}$ This can be explained by the small and superficial nature of the endoscopic biopsies compared with the full thickness surgical colectomy specimens, and the practice of prolonged medical treatment. Rudolph et al found that the prevalence of IC in a series of patients diagnosed preoperatively with UC rose from $0 \%$ to $29 \%$ when a single specialist gastrointestinal pathologist reviewed all colectomy specimens postoperatively. ${ }^{10}$

In recent epidemiological studies, ${ }^{64-67}$ and in one retrospective study, ${ }^{68}$ IC accounted for $5-6 \%$ of all initial diagnoses of IBD. However, rates as high as $23 \%$ have been reported in children. ${ }^{69}$

Given the difficulties in determining the presence of ulcerative or Crohn's colitis, it would be surprising if there were not interobserver variability in IC, and this issue is clearly a major concern. ${ }^{68}$ Given the lack of a universally accepted definition of IC, criteria for the diagnosis of IC inevitably differ between studies. ${ }^{64-68}$ The data suggest that during follow up, most patients with IC acquire a definite diagnosis of $\mathrm{UC}$ or $\mathrm{CD} .^{76869}$

\section{ANCILLARY TESTS STUDIED FOR DISTINCTION OF UC FROM CD, AND IC Serological markers}

The measurement of perinuclear antineutrophil cytoplasmic antibodies (P-ANCA) and anti-Saccharomyces cervisiae (ASCA) antibodies has been suggested as a method for differentiating UC from CD. ${ }^{70}{ }^{71}$ The presence of ASCA has a specificity of approximately $90 \%$ for $\mathrm{CD}$ and a positive predictive value of $88 \%$, but this test seems to be most positive in patients in whom there is no real diagnostic dilemma-for example, those with small bowel disease. In UC the presence of P-ANCA has a specificity approaching $90 \%$, but may be a better marker of large bowel disease than any specific subtype. The sensitivity of these tests is only $40-60 \%$, limiting their usefulness in IC. ${ }^{72}$ The clinical usefulness of these markers is controversial. ${ }^{73-75}$ A large prospective study by Joossens et al showed that ASCA and ANCA might be helpful to some degree in categorising IC. ${ }^{76}$ Patients with an initial diagnosis of IC who had positive serology were given a definitive diagnosis of CD or UC, respectively, more often than patients with negative serology. Thus, the combination of ASCA+/P-ANCA - was predictive of CD in $80 \%$, and the combination of ASCA-/P-ANCA+ was predictive of UC or UC-like CD in all patients. Patients with an initial diagnosis of IC and negative serology were more likely to retain the diagnosis of IC. These patients may represent an as yet undefined clinicoserological subgroup of IBD and may express not yet specified antibodies.

\section{Upper endoscopy}

Traditionally, inflammation in the upper gastrointestinal tract in children, in whom Helicobacter pylori infection is fairly uncommon, was tantamount to a diagnosis of CD. However, several recent case reports and series suggest that gastroduodenal involvement, which used to be diagnostic of CD, may also occur in UC, especially in children precolectomy. ${ }^{77-82}$ Although the presence of granulomas can support a diagnosis of $\mathrm{CD}$, severe inflammation and other abnormalities occur in the proximal gastrointestinal tract in CD and in UV.

One study in a paediatric population suggests that routine baseline upper endoscopy with gastric antral biopsies may help to distinguish between UC and CD. The finding of gastric antral granulomas may facilitate a diagnosis of CD in children whose colonoscopic findings are indeterminate or suggestive of UC. Diffuse antral $H$ pylori negative inflammation is of no value in differentiating CD from UC, and appears to be common in both. Focal antral gastritis is suggestive of, although not exclusive to, CD. ${ }^{77}$ Several other investigators confirm the occurrence of focal antral $H$ pylori negative gastritis in $\mathrm{CD} .^{83-87}$

A large retrospective study of children and adolescents confirms the high prevalence of focal gastritis among children with IBD relative to children without IBD or those with $H$ pylori infection, but also shows that focal gastritis can be found in patients with UC. ${ }^{88}$ The specificity and positive predictive value of focal gastritis for CD range from $79 \%$ to $84 \%$ and $71 \%$ to $81 \%$, respectively, in different studies. ${ }^{81} 848789$ Diffuse acute duodenitis is especially suggestive of UC. ${ }^{82}$

\section{Magnetic resonance imaging}

A study of magnetic resonance imaging in differentiating UC from CD in a paediatric population showed poor interobserver reliability. Magnetic resonance imaging interpretation of IBD did not adequately recognise CD in children. ${ }^{90} 1 \mathrm{H}$ magnetic resonance spectroscopy performed on colonic mucosal biopsies was used to differentiate UC from CD disease, and showed some promise in the classification of IC. ${ }^{91}$

\begin{tabular}{lc}
$\begin{array}{l}\text { Table } 3 \text { Examples of variable rates of pouch failure in } \\
\text { indeterminate colitis (IC) }\end{array}$ & $\begin{array}{l}\text { Pouch failure in IC } \\
\text { patients }\end{array}$ \\
\hline Study & $3.4 \%$ \\
\hline Delaney et al (Cleveland Clinic Foundation, & \\
USA) & $19 \%$ \\
Mclntyre et al (Mayo Clinic, USA) & $0 \%$ \\
Rudolph et al (Louisville, Kentucky, USA) & $28 \%$ \\
Yu et al (Mayo Clinic, USA) & $12 \%$ \\
Marcello et al (Labey Hitchcock Medical \\
Center, Burlington, Massachusetts, USA)
\end{tabular}

Table 3 Examples of variable rates of pouch failure in 


\section{IC AND IPAA}

Whether IPAA is the operation of choice for IC is controversial..$^{92}{ }^{93}$ Studies from the Mayo Clinic, USA, show the success rate in patients with IC who underwent IPAA to be $73-85 \%$, as compared with a success rate of $89 \%$ in definite UC. ${ }^{94-96}$ The frequency of severe pouch complications overall appears to be $20 \%$-intermediate between UC (8-10\%) and CD $(30-40 \%)$. Although this intermediate frequency of pouch complications may superficially reflect on IC as the "intermediate" form of chronic idiopathic IBD, the interpretation of these finding is not straightforward. In one series of patients with IBD who had undergone IPAA, Yu and colleagues found that $2 \%$ of patients with UC and $15 \%$ with IC ultimately had the diagnosis changed to $\mathrm{CD} .{ }^{96}$ When the outcomes of the patients with CD were considered separately, the complication rate of the remaining patients with IC was identical to that of those with UC. McIntyre et al compared functional outcomes, frequency of bowel movements, incontinence, or prevalence of pouchitis, in addition to pouch failure rate, in typical UC and in patients with IC. ${ }^{95}$ The failure rate in IC was higher than in UC ( $19 \% v 8 \%)$. However, most patients with IC had longterm functional results similar to those of patients with UC. Such findings raise the suspicion that most patients with IC probably have UC, or perhaps more accurately may be non-CD. Pouch failure rates were higher as a result of perineal disease in the IC group in McIntyre's series (presumably related to $\mathrm{CD}) .{ }^{95}$ This supports the argument that the overall higher rate of severe pouch complications in patients with IC compared with those with UC who had undergone IPAA results from the heterogeneity of the patients included in the IC group in the different series, such that they frequently represent an admixture of patients with true UC and CD. The functional outcomes of patients with UC and IC after IPAA were found to be similar in other studies. ${ }^{10997}$

"In general, we do not regard indeterminate colitis a contraindication for a pouch"

Patients with IC and IPAA manifest more late complications, such as more episodes of pelvic sepsis, pouch fistula, and pouch failure compared with patients with definite UC. ${ }^{10} 9698$

The incidence of pouch failure in patients with IC is variable among different series (table 3).

No patient with IC required permanent ileostomy in the series by Rudolph et al. ${ }^{10}$ This contrasts with the Labey and the Mayo experiences; these centres reported pouch failure in $28 \%, 16-19 \%$, and $12 \%$ of patients with IC, respectively. ${ }^{95} 969899$

The most common problem is pouchitis, which increases in incidence as the duration of follow up increases. ${ }^{94} 9596100$ The caveat in interpretation of data regarding functional outcome and complications of IPAA in patients with IC is that the level of diligence exercised by surgeons, gastroenterologists, and pathologists in attempting to classify the colitis before IPAA may vary among different series, and may contribute to some of the conflicting results seen. Gramlich et al proposed that pathological stratification of the IC colectomy specimen findings may predict those more likely to develop CD or other complications, but not pouch failure and that, on this basis, patients with IC should not be precluded from having IPAA surgery. ${ }^{101}$ In their series, postoperative colectomy diagnoses were divided into UC if there was diffuse mucosal disease involving the rectum, CD if there were granulomas or transmural lymphoid aggregates not associated with deep ulcers, and IC in the remaining specimens with atypical features. The IC group was subcategorised as IC favouring CD (segmental disease but without granulomas or transmural
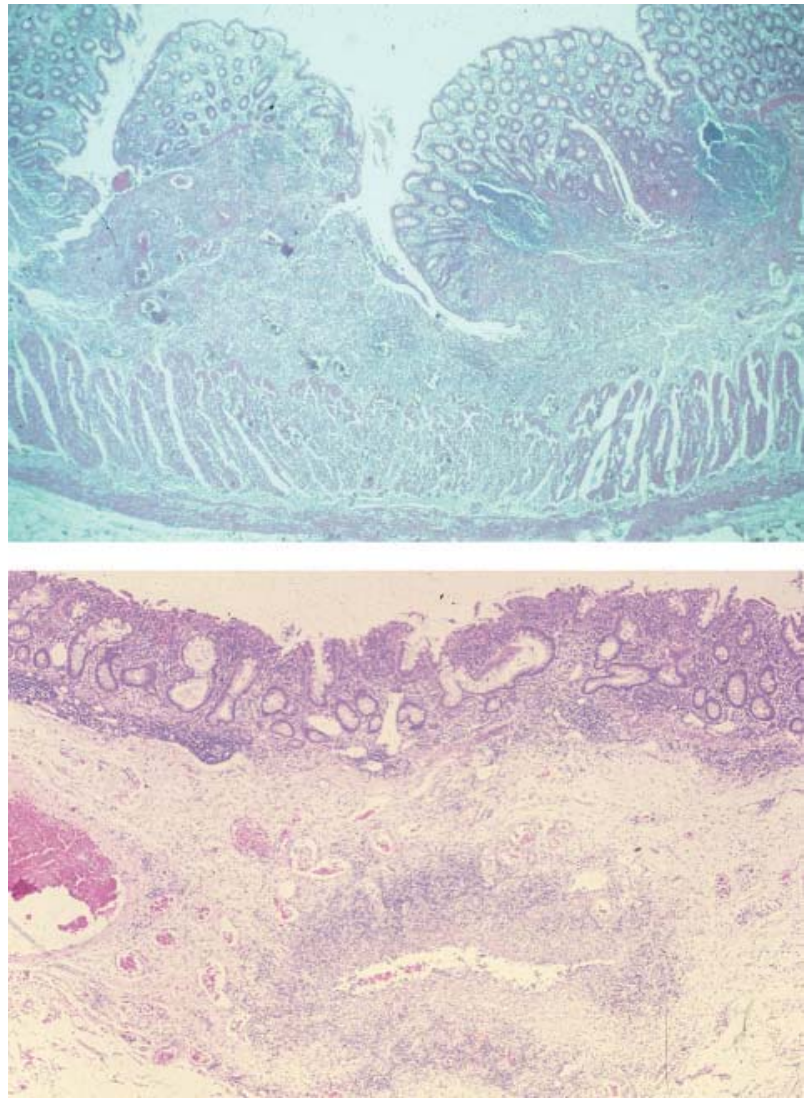

Figure 7 Undermining ulcers in fulminant colitis. (A) Note the overhanging edges. (B) Tangential sectioning of undermining edge mimics a Crohn's disease fissure (haematoxylin and eosin stain).

lymphoid aggregates not associated with deep ulcers), "true" IC, and IC favouring UC, if in addition to features of UC there was one or a combination of (1) deep ulcers, (2) transmural lymphoid aggregates, (3) skip lesions, (4) terminal ileal inflammation, and/or (5) a caecal patch. In the patients with IC favouring UC, only those with evidence of deep ulceration had a significant increase in the incidence of CD. However, it was not clear whether any or all of the patients with IC favouring UC with deep ulcers had a fulminant presentation, such that the deep ulcers may be related to the fulminant state alone. Furthermore, the incidence of $\mathrm{CD}$ in this subgroup of patients was only $4.3 \%$. The remainder of their complications (such as perianal abscess, perianal fistula, nonfunctioning pouch) may be related to factors other than deep ulcers that have not been taken into consideration, such as surgical technique and the impact of hot colitis.

In general, we do not regard IC as a contraindication for a pouch. As the level of suspicion for CD rises, our concerns are raised usually by stating-for example: "In view of the presence of ... (for example, rare subserosal lymphoid aggregates) the possibility that the underlying disease may be $\mathrm{CD}$ needs to be considered. Suggest consider delaying the restorative procedure for $6-12$ months at which time other evidence of CD should be sought if this will preclude the IPAA." This can include endoscopy through the stoma, enteroclysis, or capsule studies and upper endoscopy to look for other features of CD.

\section{SELECTED DIFFICULT SCENARIOS IN COLECTOMIES FOR IBD}

Table 4 summarises the histological features encountered in colectomy specimens and their importance. The criteria for 
subclassification of IBD, especially in the fulminant setting, are not absolute and overlap. The final conclusion with regard to $C D$ versus UC rests with the weight of evidence derived from the pattern of inflammation and a constellation of findings in a given case

In this section, we would like to offer personal observations and viewpoints, and propose an approach to some of the relatively more esoteric combinations of findings. It should be emphasised that these are opinions and personal observations that are not necessarily supported by existing studies, and as such we acknowledge that some may disagree with them.

\section{Scenario 1: ulceration in backwash ileitis}

There is no evidence in the literature that ulceration is part of the spectrum of backwash ileitis. The problem arises when the colon shows features compatible with UC, but the terminal ileum is involved by what appears to be backwash ileitis, except that it is accompanied by ulceration, pyloric metaplasia, or architectural distortion. These features, although not diagnostic of $\mathrm{CD}$, are sufficient to raise the possibility of $\mathrm{CD}$, despite the lack of other evidence in the colon. Pyloric metaplasia (ulcer associated lineage) is seen frequently in ileal resections from patients with $\mathrm{CD} .{ }^{1}$ It represents a non-specific reparative reaction in intestinal ulcers, ${ }^{102}$ and is not specific for CD. In a study of adults with definite evidence of $\mathrm{CD}$, pyloric metaplasia was identified in $22.2 \%$ of the terminal ileal biopsies. A previous study with similar data showed an incidence of only $2.27 \% .{ }^{34}$ The difference could reflect the duration of the disease, the extent of pre-existing terminal ileal ulceration, the opportunity for mucosal repair before biopsy, or the thoroughness of the endoscopic sampling and/or pathological examination. Pyloric metaplasia lacks specificity for $\mathrm{CD}$, but may be a sensitive indicator of persistent ulceration with inflammation. When found in terminal ileal biopsies, it could support the diagnosis of $\mathrm{CD}$ in the appropriate clinical setting.

We believe that when this situation is encountered, a diagnosis of "IBD, CD not excluded" should be given, with a recommendation to delay IPAA and follow up for a reasonable period of time, such as one year, to allow for other evidence of CD to declare itself.

\section{Scenario 2: aphthoid-type ulcers in UC}

Occasionally, the transition from normal to abnormal mucosa at the proximal end of an involved segment of UC shows aphthoid-type ulceration, endoscopically or grossly. In UC with rectal sparing, the transition from spared rectum to the distal end of the involved segment above may have a similar appearance. The gross/endoscopic appearances in these instances may be somewhat misleading and unnecessarily bias towards $\mathrm{CD}$. If there is no other evidence to suggest $\mathrm{CD}$, the diagnosis should still be UC or consistent with UC, especially if severely active/fulminant disease has been present.

\section{Scenario 3: proximal skip lesions in UC}

Infrequently, one encounters in a colectomy specimen distal disease with features of UC, whereby the upper margin of the diseased distal mucosa may be separated from additional more proximal diseased mucosa by a grossly skipped area. The importance of the disease distribution in this scenario is not clear. One approach would be to "ignore" this atypical UC distribution if there is no other compelling evidence of $C D$, but at least not hasten into a firm diagnosis of CD.

When an area of caecal inflammation is present, the differential diagnosis of caecal involvement separated from disease of the distal colon or rectum by normal mucosa is UC with caecal patch versus CD with a skip area. It is important to ascertain that the intervening mucosa is uninvolved, and if so not to assume that the disease is CD. Sampling of the terminal ileum is helpful, because it is usually uninvolved in UC with a caecal patch. If biopsies of the terminal ileum and intervening colonic mucosa are available and unremarkable, a diagnosis of IBD consistent with UC is made. However, if terminal ileal and intervening colonic mucosal biopsies are not available, a diagnosis of IBD is made but importantly without favouring CD. The clinician is advised to obtain such biopsies in the future and to confirm or exclude the presence of other features of CD, such as small bowel involvement and perianal disease.

\section{Scenario 4: scant deep lymphoid aggregates and "early" (small) granulomas}

Although granulomas and deeply situated lymphoid follicles appear to be the most reliable features for the diagnosis of CD

Table 4 Histological features in colectomy specimens and their importance

\begin{tabular}{|c|c|c|}
\hline Histological feature & Definition & Importance \\
\hline Focal LP inflammation & $\begin{array}{l}\text { Patchy increase of mononuclear cells in LP separated } \\
\text { by areas of normal LP cellularity }\end{array}$ & $\begin{array}{l}\text { In UC can be seen after treatment, may be seen in infection; characteristic } \\
\text { of CD especially if intense; may be seen in infection but tends to affect mid } \\
\text { and upper crypts and surface epithelium; not helpful in fulminant colitis }\end{array}$ \\
\hline Diffuse cryptitis & $\begin{array}{l}\text { Evenly distributed inflammation of crypts, involving } \\
\text { most crypts }\end{array}$ & $\begin{array}{l}\text { Characteristic of UC, seen in pretreatment material and flares of disease. } \\
\text { Avidity of neutrophils for crypts, unlike CD in biopsies and fulminant } \\
\text { colectomy specimens }\end{array}$ \\
\hline Idiopathic granulomas & $\begin{array}{l}\text { Discrete aggregate of macrophages, more than } 5 \\
\text { histiocytes }\end{array}$ & $\begin{array}{l}\text { Against a diagnosis of } U C \text { unless crypt related; characteristic of } C D \text {, but } \\
\text { seen in some infections (TB, yersinia, others); in fulminant colitis against a } \\
\text { diagnosis of UC, favours } C D\end{array}$ \\
\hline $\begin{array}{l}\text { Microgranulomas (small } \\
\text { aggregates) }\end{array}$ & $\begin{array}{l}\text { A granuloma is defined as } 5 \text { histiocytes or more and } \\
\text { everything else is not a granuloma, so microgranuloma } \\
\text { probably does not exist }\end{array}$ & Some believe carries weight in favour of CD \\
\hline $\begin{array}{l}\text { Crypt related granulomas } \\
\text { or giant cells/mucin } \\
\text { granulomas }\end{array}$ & $\begin{array}{l}\text { Giant cells or granulomatous reaction adjacent to a } \\
\text { ruptured or injured crypt }\end{array}$ & No special importance \\
\hline $\begin{array}{l}\text { Muscularis mucosa } \\
\text { duplication/hyperplasia }\end{array}$ & $\begin{array}{l}\text { Splaying, thickening, and disorganisation of smooth } \\
\text { muscle, sometimes blending with submucosa }\end{array}$ & $\begin{array}{l}\text { Indicates previous ulceration; usually seen in CD; may be seen in UC after } \\
\text { immunosuppressive treatment for temporisation of severe disease, not } \\
\text { helpful in fulminant colitis }\end{array}$ \\
\hline Narrow fissures & Cleff-like fissure extending into submucosa & $\begin{array}{l}\text { Characteristic of CD, but are tracts - usually histiocytes - at periphery } \\
\text { and neutrophils centrally (different from fissures seen in IC); may occur in } \\
\text { IC and occasionally in UC }\end{array}$ \\
\hline $\begin{array}{l}\text { Wide fissures, deep } \\
\text { undermining ulcers (fig 7A) }\end{array}$ & $\begin{array}{l}\text { V shaped clefts that may extend into submucosa or } \\
\text { muscularis propria with overhanging edges }\end{array}$ & $\begin{array}{l}\text { Typical for any form of fulminant colitis; typical of all severe IBD and some } \\
\text { infections. When undermining ulcers are tangentially cut, they may give } \\
\text { the false impression of linear fissures seen in CD (fig 7B) }\end{array}$ \\
\hline
\end{tabular}


in the fulminant colectomy specimen, they are not always present to the same extent. Occasionally there are only one or two lymphoid aggregates within the distal colonic wall in IBD that otherwise has features of UC. The dilemma is whether to attach any weight to such a minimal finding. Our approach is to surmise that given the minimal nature of the evidence, $C D$ is unlikely, and a diagnosis of IBD but favouring UC is rendered. Lymphoid aggregates in the subserosa can be polymorphous. Our personal view is to ignore inflammatory aggregates in the subserosa if they are polymorphousespecially ones with plasma cells-but not if purely lymphoid.

Where a small histiocytic aggregate ends and a granuloma begins is a subjective matter. Although there is a precedent in the literature that aggregates of five or more histiocytes represent granulomas, the cut off is nevertheless arbitrary. It is not clear whether the presence of small histiocytic aggregates signifies $\mathrm{CD}$ at all times. If this is the only evidence suggesting CD, a diagnosis of IC favouring UC is made, but one should refrain from making a definite diagnosis of $\mathrm{CD}$, and suggest delaying IPAA, with a period of follow up as outlined previously.

\section{"Although there is a precedent in the literature that aggregates of five or more histiocytes represent granulo- mas, the cut off is nevertheless arbitrary"}

With regard to ancillary tests, we personally do not use ANCA/ASCA to influence the pathological diagnosis when faced with the above scenarios or with other difficulties. This approach has the potential of combining the shortcomings of histological parameters with uncertainties of ANCA/ASCA themselves. However, if enteroclysis in a given patient shows —for example, jejunal CD-that would stop us from making a diagnosis of UC in colonic biopsies of that patient.

We do not use the term IC when faced with difficulties in classifying IBD into CD or UC on biopsy, but prefer to reserve it for colectomy specimens. We regard colectomy specimens as the court of final appeal for the histological classification of colonic IBD. We accept that the designation IC in a colectomy specimen in a given patient may serve as the working clinical diagnosis indefinitely, or the working clinical diagnosis may change to UC or CD if and when additional evidence for one or the other becomes available with follow up-the "pending tray" concept.

We prefer to use the term "IBD, not yet classified" when faced with difficulty in classifying IBD into CD or UC on biopsy. Although some may regard this as semantic nuance, we feel such a designation leaves the door open for biopsy classification of the IBD in repeat or future biopsies. These may provide more comprehensive sampling, or capture more characteristic features as the IBD evolves. The difference is that, after colectomy, there is no further opportunity for examining additional material from the colon, so if the IBD is not classifiable pathologically at that point it is then indeterminate as far as the pathological evidence in the colon goes.

\section{SUMMARY}

It appears that the jury is still out as to whether IC is a separate entity or a "third" type of IBD, or whether all are basically a form of either UC or CD. If one accepts the notion that the colon has a limited repertoire of response to injury, then IC may simply represent the end stage of any colitisUC, CD, and other diseases including pseudomembranous colitis, or other infections, because only $50 \%$ of acute self limited colitis ever has an organism identified. However, the clinical evolution of IC indicates that based on follow up investigations and clinical events it is possible to reclassify a proportion of cases into UC or CD. Price ${ }^{7}$ was able to reclassify half of his 30 cases of IC after reviewing additional material from precolectomy biopsies or from material that subsequently became available. If reclassification of IC then rests with cumulative evidence derived from evolving clinical symptoms and physical findings, imaging, endoscopy, colectomy specimens, and the pathological examination of endoscopic precolectomy and postcolectomy biopsies, IC may not be a separate entity but a provisional diagnosis. Price $^{103}$ had proposed that the term should be used as a pending tray diagnosis, representing diagnostic inadequacy, and not as a specific nosological entity. Evidence emerging from the studies of serological markers (ASCA and P-ANCA) suggests that perhaps a subgroup of patients initially diagnosed as IC may be identified as a separate group based on their distinct profile of ASCA and ANCA. Approximately $50 \%$ of patients with IC in the series of Joossens ${ }^{72}$ did not show ASCA or P-ANCA. Most of these patients retained the diagnosis of IC during their further clinical course, perhaps reflecting a distinct clinicoserological entity. ASCA and P-ANCA estimation has been suggested as a method for differentiating UC from CD, but sensitivity is only $40-60 \%$, limiting its clinical usefulness.

Some hold the view that distinguishing IC as a separate entity has clinical value because of the higher incidence of pouch complications and the greater risk of pouch failure. Regardless of whether one accepts IC as a separate entity, there are instances when there is a real need to classify the IBD-for example when deciding on specific medical treatments/trials, type of surgery (partial versus total colectomy), and the formation of pouches. These are really nodes in the management algorithm where one may need to make a firm decision, but what is most important is not whether the IC is really $\mathrm{UC}$ or $\mathrm{CD}$, but whether there are any features of $\mathrm{CD}$ that would preclude a specific type of treatment-CD versus IC/UC-given that IC seems to behave more like UC than CD for most of these nodes.

\section{"We regard colectomy specimens as the court of final appeal for the histological classification of colonic inflammatory bowel disease"}

Pathologists need to understand the different clinical pathological scenarios that lead to a diagnosis of IC, and perhaps agree to apply the term only as Price described it originally-in colectomy specimens not biopsies. This is because biopsies are fraught with pitfalls, such as the effects of treatment, and biopsies taken from large areas of the colon may be labelled as coming from one site, resulting in a histological pattern that is not typical of either UC or CD, and making the diagnosis of IC tempting. The histological diagnostic criteria for classification of IBD seem to have dulled in recent years when applied to post treatment material. Pathologists should also familiarise themselves with the features of fulminant disease, so that they are not erroneously swayed to a diagnosis of CD or IC in colectomy specimen evaluation.

There are numerous other confounding issues including the effects of treatment and the presence of caecal (and appendiceal) inflammation, which may cause the observer to consider $\mathrm{CD}$ in a patient who otherwise has a typical left sided colitis, and which may result in doubt about the diagnosis of UC and lead to a diagnosis of IC or even CD.

The terminology used in the diagnosis of biopsies and colectomy specimens should reflect the degree of certainty, or lack thereof, as to whether the underlying disease is IBD in the first place, and whether it is CD or UC. When the type of 


\section{Take home messages}

- The settings that give rise to the diagnosis of indeterminate colitis (IC) include confounding histological features of inflammatory bowel disease (IBD) in the fulminant or refractory phase, IBD in the chronic phase, the effects of treatment on the histology of IBD, unusual patterns of ulcerative colitis (UC), observer bias, colitis not caused by UC or Crohn's disease (CD), and non-specific fulminant colitis

- The distinction between $C D$ and UC rests with the weight of evidence derived from a pattern of inflammation and a constellation of findings in a given case

- It is preferable to reserve the term IC for colectomy specimens

- When faced with difficulties in classifying IBD into CD or UC in biopsies, the term IC should not be used. We prefer to use the term "IBD, not yet classified" or "colitis of uncertain aetiology". This designation leaves the door open for biopsy classification of IBD in repeat or future biopsies or resections, or from other clinical data

- The diagnosis of IC in a colectomy specimen in a given patient may stand as the clinical diagnosis indefinitely, or may change to $U C$ or $C D$ if and when additional evidence for one or the other becomes available with follow up

- Serological markers, anti-Saccharomyces cervisiae and perinuclear antineutrophil cytoplasmic antibodies, have been suggested as a method for differentiating UC from CD, but their sensitivity is only $40-60 \%$, limiting their clinical usefulness. They tend to be noncontributory when most needed

- IC should not be regarded as a contraindication for ileal pouch anal anastomosis (IPAA). The overall higher rate of severe pouch complications in patients with IC compared with those with UC and IPAA may result from the heterogeneity of patients designated as having IC in different series, such that they frequently represent an admixture of patients with $U C$ and $C D$

IBD in biopsy specimens is uncertain, it is better to call it "IBD of uncertain subtype" or "IBD not yet classified", and if uncertain of IBD, a descriptive diagnosis may be appropriate.

\section{Authors' affiliations}

M Guindi, Department of Laboratory Medicine and Pathobiology, University of Toronto, and Department of Pathology, University Health Network, Toronto, Ontario, Canada, M5G 2C4

R H Riddell, Department of Pathology, Mount Sinai Hospital, Toronto, Ontario, Canada

\section{REFERENCES}

1 Lewin KJ, Riddell RH, Weinstein WM. Gastrointestinal pathology and its clinical implications. New York: Igaku-Shoin, 1992.

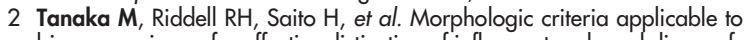
biopsy specimens for effective distinction of inflammatory bowel disease from other forms of colitis and of Crohn's disease from ulcerative colitis. Scand J Gastroenterol 1999;34:55-67.

3 Riddell RH. Histopathology of ulcerative colitis. In: Allan RN, Rhodes JM Hanaver SB, et al, eds. Inflammatory bowel disease, 3rd ed. New York: Churchill-Livingstone, 1997:291-309.

4 Goldblum JK, Petras RE. Histopathology of Crohn's disease. In: Allan RN, Rhodes JM, Hanauer SB, et al, eds. Histopathology of Crohn's disease, 3rd ed. New York: Churchill-Livingstone, 1997:311-15.
5 Schachter $\mathrm{H}$, Kirsner JB. Definitions of inflammatory bowel disease of unknown etiology. Gastroenterology 1975;68:591-600.

6 Price AB. Overlap in the spectrum of non-specific inflammatory bowel disease: colitis indeterminate. J Clin Pathol 1978;31:567-77.

7 Wells AD, McMillan I, Price AB, et al. Natural history of indeterminate colitis. Br J Surg 1991;78:179-81.

8 Lee KS, Medline A, Shockey S. Indeterminate colitis in the spectrum of inflammatory bowel disease. Arch Pathol Lab Med 1979;103:173-6.

9 Nicholls RJ, Wells AD. Indeterminate colitis. Baillieres Clin Gastroenterol 1992;6:105-12.

10 Rudolph WG, Uthoff $S M, M c A u l i f f e ~ T L$, et al. Indeterminate colitis: the real story. Dis Colon Rectum 2002;45:1528-34.

11 Swan NC, Geoghegan JG, O'Donoghue DP, et al. Fulminant colitis in inflammatory bowel disease: detailed pathologic and clinical analysis. Dis Colon Rectum 1998;41:1511-15.

12 Mahadeva U, Martin JP, Patel NK, et al. Granulomatous ulcerative colitis: a re-appraisal of the mucosal granuloma in the distinction of Crohn's disease from ulcerative colitis. Histopathology 2002;41:50-5.

13 Lee FD, Maguire C, Obeidat W, et al. Importance of cryptolytic lesions and pericryptal granulomas in inflammatory bowel disease. $J$ Clin Pathol 1997; 50:148-52.

14 Bernstein CN, Shanahan F, Anton PA, et al. Patchiness of mucosal inflammation in treated ulcerative colitis: a prospective study. Gastrointest Endosc 1995; 12:232-7.

15 Bernstein CN, Shanahan F, Weinstein WM. Histological patchiness and sparing of the rectum in ulcerative colitis: refuting the dogma. J Clin Pathol 1997;50:354-5.

16 Kleer CG, Appelman HD. Ulcerative colitis: patterns of involvement with colorectal biopsies and changes with time. Am I Surg Pathol 1998;22:983-9.

17 Levine TS, Tzardi M, Mitchell S, et al. Diagnostic difficulty arising from rectal recovery in ulcerative colitis. J Clin Pathol 1996;49:319-23.

18 Spiliadis CA, Lennard-Jones JE. Ulcerative colitis with relative sparing of the rectum. Clinical features, histology, and prognosis. Dis Colon Rectum 1987;30:334-6.

19 Hamilton SR. Diagnosis comparison of ulcerative colitis and Crohn's disease involving the colon. In: Norris HT, ed. Pathology of the colon, small intestine and anus. New York: Churchill Livingstone, 1983:1-19.

20 Hamilton SR. The differential diagnosis of idiopathic inflammatory disease by colorectal biopsy. Int J Colorectal Dis 1987;2:113-17.

21 Greenson J, Odze R. Inflammatory bowel disease of the large intestine. In: Odze RD, Goldblum JR, Crawford JM, eds. Surgical pathology of the GI tract, liver, biliary tract, and pancreas. Philadelphia, Pennsylvania: Saunders, 2004:216-17.

22 D'Haens G, Geboes K, Peeters M, et al. Patchy cecal inflammation associated with distal ulcerative colitis: a prospective endoscopic study. Am J Gastroenterol 1997;92:1275-9.

23 Okawa K, Aoki T, Sano K, et al. Ulcerative colitis with skip lesions at the mouth of the appendix. Am J Gastroenterol 1998;93:2405-10.

24 Yang SK, Jung HY, Kang GH, et al. Appendiceal orifice inflammation as a skip lesion in ulcerative colitis: an analysis in relation to medical therapy and disease extent. Gastrointest Endosc 1999:49:743-77.

25 Mutinga M, Farraye $F$, Wang $H$, et al. Clinical significance of right colonic inflammation in patients with left sided chronic ulcerative colitis [abstract]. Gastroenterology 2001;120:A450.

26 Davison AM, Dixon MF. The appendix as a "skip lesion" in ulcerative colitis. Histopathology 1990;16:93-5.

27 Groisman GM, George J, Harpaz N. Ulcerative appendicitis in universal and nonuniversal ulcerative colitis. Mod Pathol 1994;7:322-35.

28 Saltzstein SL, Rosenberg BF. Ulcerative colitis of the ileum, and regional enteritis of the colon; a comparative histological study. Am J Clin Pathol 1963;40:610-23.

29 Gustavsson S, Weiland LH, Kelly KA. Relationship of backwash ileitis to ileal pouchitis after ileal pouch-anal anastomosis. Dis Colon Rectum 1987:30:25-8.

30 Schmidt CM, Lazenby AJ, Hendrickson RJ, et al. Preoperative terminal ileal and colonic resection histopathology predicts risk of pouchitis in patients after ileoanal pull-through procedure. Ann Surg 1998;227:654-62.

31 Kim B, Barnett JL, Kleer CG, et al. Endoscopic and histological patchiness in treated ulcerative colitis. Am J Gastroenterol 1999;94:3258-62.

32 Odze R, Antonioli D, Peppercorn M, et al. Effects of topical 5-aminosalicylic acid (5-ASA) therapy on rectal mucosal biopsy morphology in chronic ulcerative colitis. Am J Surg Pathol 1993;17:869-75.

33 Geboes K, Dalle I. Influence of treatment on morphological features of mucosal inflammation. Gut 2002;50(suppl 3):I137-42.

34 Geboes K, Ectors N, D'Haens G, et al. Is ileoscopy with biopsy worthwhile in patients presenting with symptoms of inflammatory bowel disease? Am J Gastroenterol 1998;93:201-6.

35 Driman DK, Preiksaitis HG. Colorectal inflammation and increased cell proliferation associated with oral sodium phosphate bowel preparation solution. Hum Pathol 1998;29:972-8.

36 Schumacher G, Kollberg B, Sandstedt B. A prospective study of first attacks of inflammatory bowel disease and infectious colitis. Histologic course during the 1st year after presentation. Scand J Gastroenterol 1994;29:318-32.

37 Washington K, Greenson JK, Montgomery E, et al. Histopathology of ulcerative colitis in initial rectal biopsy in children. Am J Surg Pathol 2002;26:1441-9.

38 Tang LH, Reyes-Mugica M, Hao L, et al. Histologic differences between children and adults presenting with ulcerative colitis [abstract]. Mod Pathol 2000;14:96A. 
39 Markowitz J, Kahn E, Grancher K, et al. Atypical rectosigmoid histology in children with newly diagnosed ulcerative colitis. Am J Gastroenterol 1993;88:2034-7.

40 Glickman J, Bousvaros A, Farraye F. Relative rectal sparing and skip lesions are not uncommon at initial presentation in pediatric patients with chronic ulcerative colitis [abstract]. Mod Pathol 2002;15:127A.

41 Glickman J, Bousvaros A, Farraye F. Relative rectal sparing and skip lesions are not uncommon at initial presentation in pediatric patients with chronic ulcerative colitis [abstract]. Mod Pathol 2002;15:127A.

42 Moum B, Ekbom A, Vatn MH, et al. Inflammatory bowel disease: reevaluation of the diagnosis in a prospective population based study in south eastern Norway. Gut 1997;40:328-32.

43 Evans JM, McMahon AD, Murray FE, et al. Non-steroidal anti-inflammatory drugs are associated with emergency admission to hospital for colitis due to inflammatory bowel disease. Gut 1997;40:619-22.

44 Faucheron JL, Parc R. Non-steroidal anti-inflammatory drug-induced colitis. Int J Colorectal Dis 1996;11:99-101.

45 Tanaka M, Riddell RH. The pathological diagnosis and differential diagnosis of Crohn's disease. Hepatogastroenterology 1990;37:18-31.

46 Kumar NB, Nostrant TT, Appelman HD. The histopathologic spectrum of acute self-limited colitis (acute infectious-type colitis). Am J Surg Pathol 1982;6:523-9.

47 Surawicz CM, Belic L. Rectal biopsy helps to distinguish acute self-limited colitis from idiopathic inflammatory bowel disease. Gastroenterology 1984;86:104-13.

48 Le Berre N, Heresbach D, Kerbaol M, et al. Histological discrimination of idiopathic inflammatory bowel disease from other types of colitis. J Clin Pathol 1995:48:749-53.

49 Makapugay LM, Dean PJ. Diverticular disease-associated chronic colitis. Am J Surg Pathol 1996;20:94-102.

50 Shepherd NA. Diverticular disease and chronic idiopathic inflammatory bowel disease: associations and masquerades. Gut 1996;38:801-2.

51 Goldstein NS, Leon-Armin C, Mani A. Crohn's colitis-like changes in sigmoid diverticulitis specimens is usually an idiosyncratic inflammatory response to the diverticulosis rather than Crohn's colitis. Am J Surg Patho 2000;24:668-75.

52 Gledhill A, Dixon MF. Crohn's-like reaction in diverticular disease. Gut 1998:42:392-5.

53 Burroughs SH, Bowrey DJ, Morris-Stiff GJ, et al. Granulomatous inflammation in sigmoid diverticulitis: two diseases or one? Histopathology 1998;33:349-53.

54 Gupta J, Shepherd NA. Colorectal mass lesions masquerading as chronic inflammatory bowel disease on mucosal biopsy. Histopathology 2003;42:476-81.

55 Tendler DA, Aboudola S, Zacks JF, et al. Prolapsing mucosal polyps: an underrecognized form of colonic polyp-a clinicopathological study of 15 cases. Am J Gastroenterol 2002;97:370-6.

56 Gurudu S, Fiocchi C, Katz JA. Inflammatory bowel disease. Best Pract Res Clin Gastroenterol 2002;16:77-90.

57 Brandt L, Boley S, Goldberg L, ef al. Colitis in the elderly. A reappraisal. Am J Gastroenterol 1981;76:239-45.

58 Bharucha AE, Tremaine WJ, Johnson CD, et al. Ischemic proctosigmoiditis. Am J Gastroenterol 1996:91:2305-9.

59 Tsang P, Rotterdam H. Biopsy diagnosis of colitis: possibilities and pitfalls. Am J Surg Pathol 1999;23:423-30.

60 Theodossi A, Spiegelhalter DJ, Jass J, et al. Observer variation and discriminatory value of biopsy features in inflammatory bowel disease. Gut 1994;35:961-8

61 Farmer M, Petras RE, Hunt LE, et al. The importance of diagnostic accuracy in colonic inflammatory bowel disease. Am J Gastroenterol 2000;95:3184-8

62 Bentley $E$, Jenkins D, Campbell F, et al. How could pathologists improve the initial diagnosis of colitis? Evidence from an international workshop. J Clin Pathol 2002;55:955-60.

63 Kangas E, Matikainen M, Mattila J. Is "indeterminate colitis" Crohn's disease in the long-term follow-up? Int Surg 1994;79:120-3

64 Shivananda S, Lennard-Jones J, Logan R, et al. Incidence of inflammatory bowel disease across Europe: is there a difference between north and south? Results of the European collaborative study on inflammatory bowel disease (EC-IBD). Gut 1996;39:690-7

65 Moum B, Vatn MH, Ekbom A, et al. Incidence of ulcerative colitis and indeterminate colitis in four counties of southeastern Norway, 1990-93-a prospective population-based study. Scand J Gastroenterol 1996;31:362-6.

66 Martinez-Salmeron JF, Rodrigo M, de Teresa J, et al. Epidemiology of inflammatory bowel disease in the province of Granada, Spain: a retrospective study from 1979 to 1988. Gut 1993:34:1207-9.

67 Russel MGVM, Dorant E, Volovics A, et al. High incidence of inflammatory bowel disease in the Netherlands - results of a prospective study. Dis Colon Rectum 1998;41:33-40.

68 Meucci G, Bortoli A, Riccioli FA, et al. Frequency and clinical evolution of indeterminate colitis: a retrospective multi-centre study in northern ltaly. GSMII (Gruppo di Studio per le Malattie Infiammatorie Intestinali). Eur J Gastroenterol Hepatol 1999; 1 1:909-13.

69 Hildebrand H, Fredrikzon B, Holmquist L, et al. Chronic inflammatory bowel disease in children and adolescents in Sweden. J Pediatr Gastroenterol Nutr 1991; 13:293-7.

70 Quinton JF, Sendid B, Reumaux D, et al. Anti-Saccharomyces cerevisiae mannan antibodies combined with antineutrophil cytoplasmic autoantibodies in inflammatory bowel disease: prevalence and diagnostic role. Gut 1998:42:788-91.

71 Zachos M, Reumaux D, Critch J, et al. Clinical utility of ASCA and ANCA in pediatric colitis. J Pediatr Gastroenterol Nutr 2001;33:378-81.

72 Legnani PE, Kornbluth A. Difficult differential diagnoses in IBD: ileitis and indeterminate colitis. Semin Gastrointest Dis 2001;12:211-22.

73 Targan SR. The utility of ANCA and ASCA in inflammatory bowel disease. Inflamm Bowel Dis 1999:5:61-3.

74 MacDermott RP. Lack of current clinical value of serological testing in the evaluation of patients with IBD. Inflamm Bowel Dis 1999:5:64-5.

75 Present DH, Banks PA. The role of PANCA and ASCA in differentiating ulcerative colitis, Crohn's disease, and indeterminate colitis. Inflamm Bowel Dis 1999:5:66-7.

76 Joossens S, Reinisch W, Vermeire S, et al. The value of serologic markers in indeterminate colitis: a prospective follow-up study. Gastroenterology 2002; 122:1242-7.

77 Tobin JM, Sinha B, Ramani P, et al. Upper gastrointestinal mucosal disease in pediatric Crohn's disease and ulcerative colitis: a blinded, controlled study. J Pediatr Gastroenterol Nutr 2001;32:443-8.

78 Kaufman SS, Vanderhoof JA, Young R, et al. Gastroenteric inflammation in children with ulcerative colitis. Am J Gastroenterol 1997;92:1209-12.

79 Sasaki M, Okada K, Koyama S, et al. Ulcerative colitis complicated by gastroduodenal lesions. J Gastroenterol 1996:31:585-9.

80 Honma J, Mitomi H, Murakami K, et al. Nodular duodenitis involving CD8+ cell infiltration in patients with ulcerative colitis. Hepatogastroenterology 2001;48:1604-10.

81 Kundhal PS, Stormon MO, Zachos $M$, et al. Gastral antral biopsy in the differentiation of pediatric colitides. Am J Gastroenterol 2003;98:557-61.

82 Valdez R, Appelman HD, Bronner MP, et al. Diffuse duodenitis associated with ulcerative colitis. Am J Surg Pathol 2000;24:1407-13.

83 Schmitz-Moormann P, Malchow H, Pittner PM. Endoscopic and bioptic study of the upper gastrointestinal tract in Crohn's disease patients. Pathol Res Pract 1985; 179:377-87.

84 Parente F, Cucino C, Bollani S, et al. Focal gastric inflammatory infiltrates in inflammatory bowel diseases: prevalence, immunohistochemical characteristics, and diagnostic role. Am J Gastroenterol 2000:95:705-11.

85 Wright CL, Riddell RH. Histology of the stomach and duodenum in Crohn's disease. Am J Surg Pathol 1998;22:383-90.

86 Meining A, Bayerdörffer E, Bästlein E, et al. Focal inflammatory infiltrations in gastric biopsy specimens are suggestive of Crohn's disease. Scand J Gastroenterol 1997;32:813-18.

87 Oberhuber G, Puspok A, Oesterreicher C, et al. Focally enhanced gastritis: a frequent type of gastritis in patients with Crohn's disease. Gastroenterology 1997:112:698-706.

88 Sharif F, McDermott M, Dillon $M$, et al. Focally enhanced gastritis in children with Crohn's disease and ulcerative colitis. Am J Gastroenterol 2002;97:1415-20.

89 Oberhuber G, Hirsch M, Stolte M. High incidence of upper gastrointestina tract involvement in Crohn's disease. Virchows Arch 1998;432:49-52.

90 Durno CA, Sherman P, Williams T, et al. Magnetic resonance imaging to distinguish the type and severity of pediatric inflammatory bowel diseases. J Pediatr Gastroenterol Nutr 2000;30:170-14.

91 Bezabeh T, Somorjai RL, Smith IC, et al. The use of $1 \mathrm{H}$ magnetic resonance spectroscopy in inflammatory bowel diseases: distinguishing ulcerative colitis from Crohn's disease. Am J Gastroenterol 2001;96:442-8.

92 Wolff BG. Is ileoanal the proper operation for indeterminate colitis: the case for. Inflamm Bowel Dis 2002;8:362-5.

93 Schoetz DJJ. Is ileoanal the proper operation for indeterminate colitis: the case against. Inflamm Bowel Dis 2002;8:366-7.

94 Pezim ME, Pemberton JH, Beart RW, et al. Outcome of "indeterminant" colitis following ileal pouch-anal anastomosis. Dis Colon Rectum 1989;32:653-8.

95 McIntyre PB, Pemberton JH, Wolff BG, et al. Indeterminate colitis. Long-term outcome in patients after ileal pouch-anal anastomosis. Dis Colon Rectum 1995;38:51-4.

96 Yu CS, Pemberton JH, Larson D. Ileal pouch-anal anastomosis in patients with indeterminate colitis: long-term results. Dis Colon Rectum 2000;43:1487-96.

97 Delaney CP, Remzi FH, Gramlich T, et al. Equivalent function, quality of life and pouch survival rates after ileal pouch-anal anastomosis for indeterminate and ulcerative colitis. Ann Surg 2002;236:43-8.

98 Koltun WA, Schoetz DJJ, Roberts PL, et al. Indeterminate colitis predisposes to perineal complications after ileal pouch-anal anastomosis. Dis Colon Rectum 1991;34:857-60

99 Marcello PW, Schoetz DJ Jr, Roberts PL, et al. Evolutionary changes in the pathologic diagnosis after the ileoanal pouch procedure. Dis Colon Rectum 1997;40:263-9.

100 Rauh SM, Schoetz DJJ, Roberts PL, et al. Pouchitis - is it a wastebasket diagnosis? Dis Colon Rectum 1991;34:685-9.

101 Gramlich T, Delaney CP, Lynch AC, et al. Pathological subgroups may predict complications but not late failure after ileal pouch-anal anastomosis for indeterminate colitis. Colorectal Dis 2003;5:315-19.

102 Hanby AM, Wright NA. The ulcer-associated cell lineage: the gastrointestinal repair kit? J Pathol 1993;171:3-4.

103 Price AB. Indeterminate colitis-broadening the perspective. Curr Diagn Pathol 1999;3:35-44. 